\title{
Predictive Value of Procalcitonin in Acute Severe Ulcerative Colitis: Not Quite PerfeCT?
}

\author{
Deok Whan Kim ${ }^{1}$. Jeong Min An ${ }^{1}$. Seong Pyo Hong ${ }^{1} \cdot \mathrm{Ki} \mathrm{Baik} \mathrm{Hahm}^{1}$
}

Published online: 7 February 2019

(c) Springer Science+Business Media, LLC, part of Springer Nature 2019

Owing to the recent advances in drug therapy for inflammatory bowel disease (IBD), the treatment of acute severe ulcerative colitis (ASUC) has changed, with consequent remarkable decreases in the need for emergency surgery, a profound decline in mortality, and improved quality-of-life. For instance, infliximab salvage therapy is now associated with significantly lower rates of both ASUC and colectomy [1]. Though surgery remains an important option dependent on the patient's overall condition and disease stage, multidisciplinary medical management of ASUC has emerged as the dominant therapeutic modality, with biologic treatment as first-line option in the absence of surgical indications such as perforation or obstruction. Although still one-fifth of patients with UC experience an acute flare requiring either hospitalization or surgery, there is a need for a biomarker that would modify therapy and predict outcomes which in turn can inform the optimal timing and choice of interventions [2].

Among several candidates for ASUC biomarkers, procalcitonin (PCT), a 116-amino acid precursor of the 32-amino acid peptide hormone calcitonin, is a promising candidate. In contrast to the neuroendocrine origin of calcitonin, the source of PCT is of non-neuroendocrine [3]. Serum concentrations of PCT are increased in response to the entry of lipopolysaccharide of Gram-negative bacteria (comprising the major proportion of the intestinal microbiome) into the circulation, inducing inflammatory cytokines implicated in the pathogenesis of IBD through activation of Toll-like receptors [3]. In the past decade, PCT concentrations exceeding $0.5 \mu \mathrm{g} / \mathrm{L}$ have identified sepsis and the systemic inflammatory response in critically ill patients [4]. Therefore, serum PCT is a promising disease activity marker reflecting the inflammatory response in IBD. Nevertheless, in patients

Ki Baik Hahm

hahmkb@cha.ac.kr

1 Digestive Disease Center, CHA University Bundang Medical Center, 59 Yatap-ro, Bundang-gu, Seongnam, Korea with mild-moderate UC, serum PCT is $\leq 0.10 \mu \mathrm{g} / \mathrm{L}$, similar to concentrations measured in healthy volunteers [5]. Collectively summarized, in cases with PCT $>0.5 \mathrm{ng} / \mathrm{mL}$, there is a high probability of the presence of infective complications including bacterial enterocolitis, bacterial gastroenteritis, intra-abdominal abscess, postsurgical infection, and sepsis in IBD. All of these results support the contention that serum PCT is useful for monitoring IBD-associated infections and complications rather than assessing disease activity itself [6]. Yet, though a small number of subjects were studied by Lippi et al. [6], there was a significant association between the inflammatory markers erythrocyte sedimentation rate, C-reactive protein (C-RP), and WBC counts and serum PCT in patients suffering from severe UC.

In this issue of Digestive Diseases and Sciences, Wu et al. [7] correlated the association between serum PCT and short-term outcomes in patients with ASUC, concluding that serum $\mathrm{PCT} \geq 0.10 \mu \mathrm{g} / \mathrm{L}(\mathrm{OR}=4.134, p=0.001)$ predicts failure of intravenous corticosteroids (IVCS) therapy with a high specificity, and, when combined with measurement of fecal calprotectin (FC) $\geq 1500 \mu \mathrm{g} / \mathrm{g}$, the sensitivity was improved. Since serum PCT correlated significantly with the Ulcerative Colitis Endoscopic Index of Severity (UCEIS, $p<0.001)$ and FC ( $p<0.001)$, they concluded that serum PCT measured on admission could be a potential early noninvasive predictive biomarker for IVCS failure in ASUC patients and that the combination of PCT and FC could improve the predictive value, concluding that serum PCT could act as an alternative or supplementary predictive biomarker in ASUC patients.

In addition to the identified significance of serum PCT levels in predicting the need for intensive therapy in ASUC patients, PCT can also differentiate Clostridium difficilerelated colitis from colonization in UC [8] and provides additional benefit to the measurement of $\mathrm{C}$ - $\mathrm{RP}$ in assessing the severity of CD [9], discriminating irritable bowel syndrome (IBS) complicated by self-limited colitis or infectious gastroenteritis. PCT is also used as a biomarker for 
gastrointestinal disorders such as intestinal ischemia and pancreatitis and outside of gastroenterology in the assessment and monitoring of bacteremia in critical care, sepsis, venomous snakebites, vasculitis, antibiotic use, pneumonia, joint infection, and cholangitis [10]. In light of the findings of Wu et al. [7], PCT shows promise alone or in combination with more established inflammatory biomarkers in severity prediction and disease monitoring of ASUC.

\section{References}

1. Croft A, Walsh A, Doecke J, Cooley R, Howlett M, Radford-Smith G. Outcomes of salvage therapy for steroid-refractory acute severe ulcerative colitis: ciclosporin vs. infliximab. Aliment Pharmacol Ther. 2013;38:294-302.

2. Gallo G, Kotze PG, Spinelli A. Surgery in ulcerative colitis: When? How? Best Pract Res Clin Gastroenterol. 2018;32-33:71-78.

3. Biju PG, Garg S, Wang W, et al. Procalcitonin as a predictive biomarker for total body irradiation induced bacterial load and lethality in mice. Shock. 2012;38:170-176.

4. Wacker C, Prkno A, Brunkhorst FM, et al. Procalcitonin as a diagnostic marker for sepsis: a systematic review and meta-analysis. Lancet Infect Dis. 2013;13:426-435.
5. Kondo S, Ohkusa T, Tanakura K, et al. Clinical significance of serum procalcitonin in patients with ulcerative colitis. World $J$ Gastroenterol. 2013;19:8335-8341.

6. Lippi G, Sanchis-Gomar F. Procalcitonin in inflammatory bowel disease: drawbacks and opportunities. World J Gastroenterol. 2017;23:8283-8290.

7. Wu HM, Wei J, Li J, et al. Serum procalcitonin as a potential early predictor for short-term outcomes in acute severe ulcerative colitis. Dig Dis Sci. (Epub ahead of print). https://doi.org/10.1007/ s10620-018-5446-0.

8. Reinink AR, Limsrivilat J, Reutemann BA, et al. Differentiating Clostridium difficile colitis from Clostridium difficile colonization in ulcerative colitis: a role for procalcitonin. Digestion. 2017;96:207-212.

9. Oussalah A, Laurent V, Bruot O, et al. Additional benefit of procalcitonin to $\mathrm{C}$-reactive protein to assess disease activity and severity in Crohn's disease. Aliment Pharmacol Ther. 2010;32:1135-1144.

10. Thia KT, Chan ES, Ling KL, Ng WY, Jacob E, Ooi CJ. Role of procalcitonin in infectious gastroenteritis and inflammatory bowel disease. Dig Dis Sci. 2008;53:2960-2968. https://doi.org/10.1007/ s10620-008-0254-6.

Publisher's Note Springer Nature remains neutral with regard to jurisdictional claims in published maps and institutional affiliations. 